\title{
High Efficacy of Levofloxacin-Dexlansoprazole-Based Quadruple Therapy as a First Line Treatment for Helicobacter pylori Eradication in Thailand
}

\author{
Hatainuch Prapitpaiboon ${ }^{1}$, Varocha Mahachai ${ }^{2,3}$, Ratha-Korn Vilaichone ${ }^{1,3 *}$
}

\begin{abstract}
Background: Levofloxacin is an effective medication for second line Helicobacter pylori (H. pylori) eradication. However, limited studies have approved its use as an effective antibiotic in first line therapy. Dexlansoprazole is a new PPI and lacks of evidence in support of a role in $\mathrm{H}$. pylori eradication. This study was designed to evaluate efficacy of levofloxacin-dexlansoprazole-based quadruple therapy for $H$.pylori eradication in Thailand. Materials and Methods: This prospective randomized control study was performed during June 2014 to December 2014. $H$. pylori infected gastritis patients were randomized to receive 7- or 14-day levofloxacin-dexlansoprazole based on quadruple therapy (levofloxacin $500 \mathrm{mg}$ OD, dexlansoprazole $60 \mathrm{mg}$ bid, clarithromycin MR $1000 \mathrm{mg}$ OD, bismuth subsalicylate $1048 \mathrm{mg}$ bid). CYP2C19 genotyping and antibiotic susceptibility tests were conducted for all patients. A $13 \mathrm{C}$ urea breath test was performed to confirm $H$. pylori eradication at least 4 weeks after treatment. Results: A total of 100 patients were enrolled, comprising 44 males and 56 females (mean age of 52.6 years). Eradication rate by PP analysis was $85.7 \%(42 / 49)$ with the 7 -day regimen and $98 \%(48 / 49)$ with the 14-day regimen ( $85.7 \%$ vs $98 \%$; $p$-value $=0.059)$. ITT analysis was $84 \%$ and $96 \%$ with 7 - and 14-day regimens, respectively $(84 \%$ vs $96 \%$; p-value $=0.092)$. Antibiotic susceptibility testing demonstrated $35.1 \%$ resistance to metronidazole, $18.3 \%$ to clarithromycin, and $13.5 \%$ to levofloxacin. CYP2C19 genotyping revealed $54.1 \% \mathrm{RM}$, $34.7 \%$ IM and $11.2 \%$ PM. The 14-day regimen provided $100 \%$ eradication in patients with clarithromycin or dual clarithromycin and metronidazole $H$.pylori resistant strains. Moreover, the eradication rate was $96.6 \%$ in patients with CYP2C19 genotype RM. Conclusions: The 14-day levofloxacin-dexlansoprazole based quadruple therapy provides high $\boldsymbol{H}$. pylori eradication regardless of CYP2C19 genotype, clarithromycin or dual clarithromycin and metronidazole resistant strains. This regimen could be use as an alternative first line therapy for $\mathrm{H}$. pylori eradication in Thailand.
\end{abstract}

Keywords: Levofloxacin - dexlansoprazole - quadruple therapy - Helicobacter pylori eradication - Thailand

Asian Pac J Cancer Prev, 16 (10), 4353-4356

\section{Introduction}

Helicobacter pylori (H. pylori), a gram-negative bacterium found on the luminal surface of the gastric epithelium, was first isolated in 1984 (Marshall and Warren, 1984). It induces chronic inflammation of the underlying mucosa resulting in the development of important upper gastrointestinal diseases such as gastritis, peptic ulcer diseases, mucosa-associated lymphoid tissue (MALT) lymphoma, and gastric cancer (Rauws and Tytgat, 1990; Parsonnet et al., 1991; Bayerdorffer et al., 1995; Vilaichone and Mahachai, 2001). Moreover, a recent meta-analysis has shown that $H$.pylori eradication potentially reduces the incidence of gastric cancer, especially in high prevalence area of gastric cancer (Ford et al., 2014).
For years, H. pylori has been regarded as a difficultto-treat infection due to the bacterium's nature, and readily acquired resistance to commonly used antibiotics. Therefore, standard triple therapy with proton pump inhibitor (PPI), amoxicillin and clarithromycin is no longer recommended as an empiric choice in most countries (Chey and Wong, 2007; Mahachai et al., 2011). Levofloxacin-based triple therapy for $H$. pylori eradication has been shown to increase cure rate with minimal side effects. However, levofloxacin-based triple therapy provided excellent results only in levofloxacin sensitivity (Antos et al., 2006). Interestingly, recent studies have demonstrated the positive effect of adding bismuth salt to levofloxacin-based triple therapy for $H$.pylori eradication, especially in levofloxacin resistance (Liao et al., 2013).

We conduct this study for searching an optimal

${ }^{1}$ Gastroenterology Unit, Thammasat University Hospital, Pathumthani, ${ }^{2}$ Gastrointestinal and Liver Center, Bangkok Medical Center ${ }^{3}$ National Gastric Cancer and Helicobacter pylori Research Center, Bangkok, Thailand *For correspondence: Vilaichone@hotmail. co.th 
combination of drugs and duration for first line $H$.pylori eradication in Thailand. We reported a prospective randomized trial evaluating $H$. pylori eradication by using levofloxacin-dexlansoprazole based quadruple therapy for 7- or 14-day. The effects of CYP2C19 genotype and antibiotic resistance were also examined.

\section{Materials and Methods}

\section{Patients}

Eligible patients age 18-75 years who underwent gastroscopic examination at Thammasat University Hospital, for dyspeptic symptoms between June 2014 and December 2014 were enrolled. After the endoscopy, those with diagnosis of non-ulcer dyspepsia, which was established during gastroscopy with normal finding or mild gastritis, were considered for entry in this study. Exclusion criteria included patients with a history of prior H. pylori eradication, previous gastric surgery, pregnancy, lactation, major systemic diseases, cardiovascular disease or administration of antibiotics, bismuth and PPI drugs in the preceding 4 weeks, or allergy to any one of the given medication in the regimens. All patients provided written informed consent.

\section{The diagnosis of H. pylori infection}

During the endoscopy, 4 biopsy samples from gastric antrum were obtained for rapid urease test, $H$.pylori culture and Epsilometer test (E-test) or GenoType ${ }^{\circledR}$ HelicoDR, histological examination and CYP2C19 genotype. The results of CYP2C19 genotype testing were expressed as: rapid metabolizer (RM), intermediate metabolizer (IM) or poor metabolizer (PM). The presence of H. pylori was defined as: (1) positive $H$. pylori culture, or (2) positive tests (rapid urease test or histology).

\section{Therapeutic regimens}

Randomization was made by reference to a computergenerated list. The two different groups were given for 7-day or 14-day of 500mg levofloxacin once daily, $60 \mathrm{mg}$ dexlansoprazole twice daily, $1 \mathrm{~g}$ long acting clarithromycin MR once daily, and $1048 \mathrm{mg}$ bismuth subsalicylate twice daily.

\section{Post-therapy follow-up}

At least 4 weeks after completion of therapy, 13C-urea breath test (UBT) was carried out in all patients to assess $H$. pylori eradication. Successful eradication was defined as a negative result from UBT. Pill count was conducted, and drug consumption over $90 \%$ was defined as good compliance. Side effects were assessed by personal interview using open-ended questions. The potential adverse events listed in the questionnaires were diarrhea, bitter taste, nausea, vomiting, palpitation, and skin rashes. New symptoms and exacerbation of pre-existing symptoms during the treatment period were considered to be therapy-related adverse events. Serious adverse events were defined as events that disturbed on daily activities.

\section{Statistical analysis}

We expected the eradication rate of levofloxacin- dexlansoprazole based quadruple therapy as an empiric therapy to be $\geq 90 \%$. Treatment success was pre-specified as a cure rate of $\geq 95 \%$ (i.e. grade $A$ ) as described in previous studies (Graham et al., 2007), and failure as a cure rate of $<90 \%$ per protocol. The demographic characteristics and frequencies of adverse effects were compared using chi-squared, Fisher's exact and student's t-test. The p-value $<0.05$ was considered to be statistically significant. The study was conducted according to the good clinical practice guideline, as well as the Declaration of Helsinki, and was approved by our local ethics committee.

\section{Results}

Total of 100 patients were included in this study, 44 men and 56 women with a mean age of 52.6 years. All 100 patients were randomized in to 2 groups as previously described. When compared, the patients with 7-day regimens were significantly younger and predominate with women. The baseline demographic data are shown in Table 1. One patient in each group discontinued treatment due to side effects.

\section{Eradication of $H$. pylori infection}

The results analyzed by both intention-to-treat (ITT) and per-protocol (PP) analyses were demonstrated as shown in Figure 1. The eradication rates by ITT analysis was $84 \%(42 / 50)$ with 7-day regimen and $96 \%(48 / 50)$

\section{Table 1. Baseline Demographic Data of All Patients}

\begin{tabular}{lrrr}
\hline Characteristic data & $\begin{array}{c}\text { 7-day } \\
\text { regimen } \\
(\mathrm{n}=50)\end{array}$ & $\begin{array}{c}\text { 14-day } \\
\text { regimen } \\
(\mathrm{n}=50)\end{array}$ & P-value \\
\hline Age (years) & 49.72 & 55.64 & 0.008 \\
Sex no. (\%) & $16(32)$ & $28(56)$ & 0.026 \\
$\quad$ Male & $34(68)$ & $22(44)$ & \\
$\quad$ Female & & & \\
Underlying disease no. (\%) & $7(14)$ & $11(22)$ & 0.436 \\
$\quad$ Hypertension & $3(6)$ & $5(10)$ & 0.715 \\
$\quad$ Dyslipidemia & $4(8)$ & $6(12)$ & 0.741 \\
Smoking no. (\%) & $13(26)$ & $11(22)$ & 0.815 \\
Alcohol consumption no. (\%) & $13(r)$ \\
\hline
\end{tabular}

Table 2. Results of CYP2C19 Genotype and Eradication Rate

\begin{tabular}{lcc}
\hline $\begin{array}{l}\text { CYP2C19 } \\
\text { genotype } \\
(\mathrm{n}=98)\end{array}$ & $\begin{array}{c}7 \text {-day } \\
\text { regimen } \\
(\mathrm{n}=49)\end{array}$ & $\begin{array}{c}\text { 14-day } \\
\text { regimen } \\
(\mathrm{n}=49)\end{array}$ \\
\hline $\mathrm{RM}(\mathrm{n}=53 ; 54.1 \%)$ & $24(87.5 \%)$ & $29(96.6 \%)$ \\
$\mathrm{IM}(\mathrm{n}=34 ; 34.7 \%)$ & $20(85 \%)$ & $14(100 \%)$ \\
$\mathrm{PM}(\mathrm{n}=11 ; 11.2 \%)$ & $5(100 \%)$ & $6(100 \%)$ \\
\hline
\end{tabular}

Table 3. Adverse Events in Each Regimen

\begin{tabular}{lccc}
\hline Adverse events & $\begin{array}{c}\text { 7-day regimen } \\
(\mathrm{n}=50)\end{array}$ & $\begin{array}{c}\text { 14-day regimen } \\
(\mathrm{n}=50)\end{array}$ & P-value \\
\hline Bitter taste & $45(90 \%)$ & $44(88 \%)$ & 1 \\
Nausea/Vomiting & $5(10 \%)$ & $2(4 \%)$ & 0.436 \\
Diarrhea & $1(2 \%)$ & $2(4 \%)$ & 1 \\
Black stool & $47(94 \%)$ & $48(96 \%)$ & 1 \\
\hline
\end{tabular}




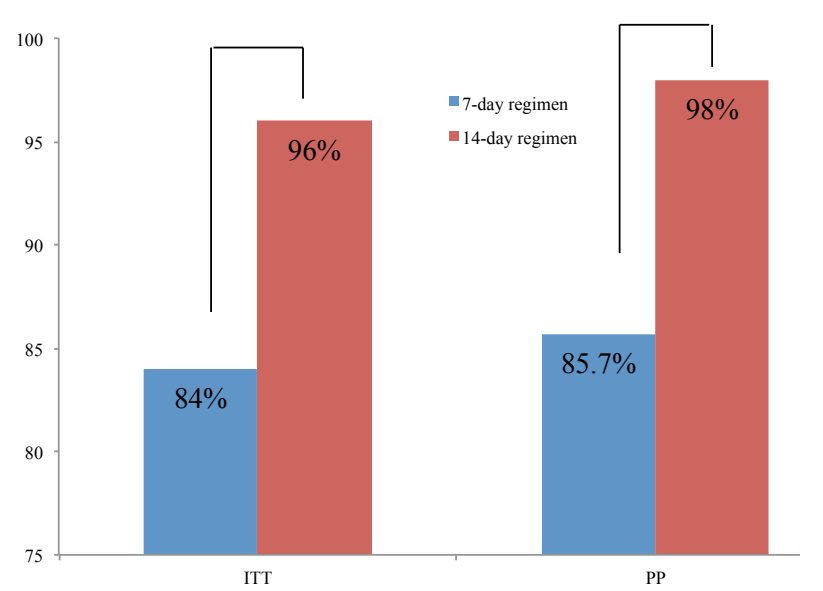

Figure 1. Eradication Rates According to Treatment Regimens

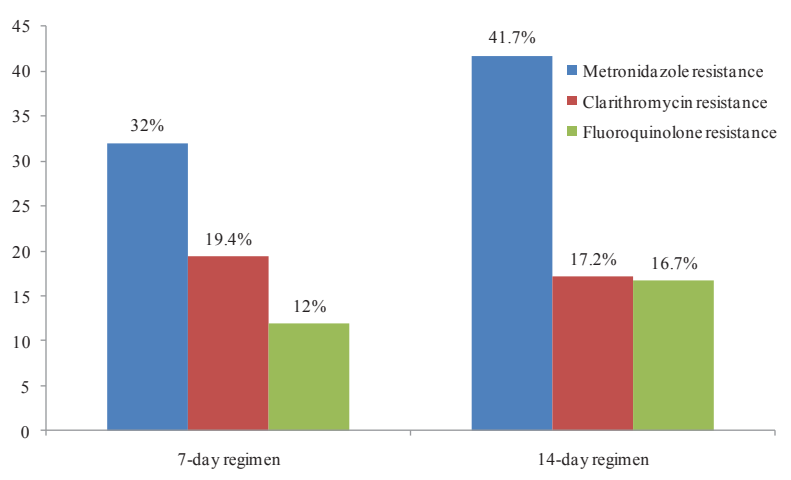

Figure 2. Prevalence of Antibiotic Resistance

with 14-day regimen. PP eradication results were $85.7 \%$ (42/49) and 98\% (48/49) with 7- and 14-day regimen, respectively. The cure rate was higher in 14-day regimen than those in 7-day regimen from both ITT and PP and could achieve cure rate of grade A $(\geq 95 \%)$ as detail in Figure 1.

Antibiotic susceptibility tests were performed in 60 strains ( 37 from E-test and 23 from GenoType ${ }^{\circledR}$ HelicoDR), which demonstrated $35.1 \%$ of metronidazole resistant, $18.3 \%$ of clarithromycin resistant and $13.5 \%$ of levofloxacin resistant strains as detail in Figure 2. CYP2C19 genotype tests were performed in 98 cases (49 from each 7-day and 14-day regimen). The CYP2C19 genotype tests revealed 54.1\% RM, 34.7\% IM and $11.2 \%$ PM. The prevalence of CYP2C19 genotype was similar in all groups of patients as in Table 2 .

\section{Adverse events}

The common side effects include diarrhea, bitter taste, nausea, and vomiting which were found in all groups. Two patients (one from each study group) were withdrawn from treatment regimens because of side effects. Documented adverse reactions are shown in Table 3 . None of subject experienced any major adverse event.

\section{Discussion}

At present, several epidemiological and experimental data support a pathological role between H.pylori and the development of gastric cancer (Basiri et al., 2014; Demirel et al., 2013; Rauws and Tytgat, 1990). It was estimated that $H$. pylori infection accounts for approximately 650,000 new cases of gastric cancer annually (de Martel et al., 2012). The eradication rate of $H$. pylori by standard triple therapy with clarithromycin-containing regimen was reported to be less than $70 \%$ in many countries worldwide, including Thailand (Vilaichone et al., 2006; Graham, 2009). It has been suggested that clarithromycincontaining regimens should be renounced as an empiric therapy especially in high clarithromycin resistance area.

Bismuth has long been known as an anti-H. pylori drug with minimal side effects. The previous study demonstrated that adding bismuth might be a good option to improve the eradication by standard triple therapy apart from increasing the dosage and duration of PPI (Srinarong et al., 2014). Several studies also demonstrated that administration of bismuth in quadruple therapy increased the eradication rate of $H$. pylori up to $90 \%$, comparing to standard triple therapy (Ford et al., 2008; Fock et al., 2009).

Fluoroquinolones have been suggested as alternative drug but generally the results of fluoroquinolone triple therapy was less than 90\% (Gisbert and Morena, 2006; Graham and Shiotani, 2012). In addition, recent studies have demonstrated that longer duration with 14-day fluoroquinolone triple therapy provided treatment success of 95\% (Miehlke et al., 2011).

Our study supported this idea and also demonstrated high eradication rate (grade A) of $H$.pylori infection from 14-day levofloxacin-dexlansoprazole quadruple therapy as a first line treatment regardless of CYP2C19 genotype, clarithromycin or dual clarithromycin and metronidazole resistant strains. Only minor side effects were observed from this regimen. Furthermore, this 14-day regimen could achieve high cure rate with levofloxacin resistant strains. However, larger multi-center controlled studies are needed to confirm this hypothesis.

\section{References}

Antos D, Schneider-Brachert W, Bastlein E, et al (2006). 7-day triple therapy of Helicobacter pylori infection with levofloxacin, amoxicillin, and high-dose esomeprazole in patients with known antimicrobial sensitivity. Helicobacter, 11, 39-45.

Basiri Z, Safaralizadeh R, Bonyadi MJ, et al (2014). Helicobacter pylori vacA d1 genotype predicts risk of gastric adenocarcinoma and peptic ulcers in northwestern Iran. Asian Pac J Cancer Prev, 15, 1575-9.

Bayerdorffer E, Neubauer A, Rudolph B, et al (1995). Regression of primary gastric lymphoma of mucosa-associated lymphoid tissue type after cure of Helicobacter pylori infection. MALT Lymphoma Study Group. Lancet, 345, 1591-94.

Chey WD, Wong BC (2007). American College of Gastroenterology guideline on the management of Helicobacter pylori infection. Am J Gastroenterol, 102, 1808-25.

de Martel C, Ferlay J, Franceschi S, et al (2012). Global burden of cancers attributable to infections in 2008: a review and synthetic analysis. Lancet Oncol, 13, 607-15.

Demirel BB, Akkas BE, Vural GU (2013). Clinical factors related with Helicobacter pylori infection--is there an association 
with gastric cancer history in first-degree family members? Asian Pac J Cancer Prev, 14, 1797-802.

Fock KM, Katelaris P, Sugano K, et al (2009). Second asiapacific consensus guidelines for Helicobacter pylori infection. J Gastroenterol Hepatol, 24, 1587-600.

Ford AC, Forman D, Hunt RH, et al (2014). Helicobacter pylori eradication therapy to prevent gastric cancer in healthy asymptomatic infected individuals: systematic review and meta-analysis of randomised controlled trials. $B M J, \mathbf{3 4 8}$, 3174.

Ford AC, Malfertheiner P, Giguere M, et al (2008). Adverse events with bismuth salts for Helicobacter pylori eradication: systematic review and meta-analysis. World J Gastroenterol, 14, 7361-70.

Gisbert JP, Morena F (2006). Systematic review and metaanalysis: levofloxacin-based rescue regimens after Helicobacter pylori treatment failure. Aliment Pharmacol Ther, 23, 35-44.

Graham DY (2009). Efficient identification and evaluation of effective Helicobacter pylori therapies. Clin Gastroenterol Hepatol, 7, 145-8.

Graham DY, Lu H, Yamaoka Y (2007). A report card to grade Helicobacter pylori therapy. Helicobacter, 12, 275-8.

Graham DY, Shiotani A (2012). Which Therapy for Helicobacter pylori Infection? Gastroenterology, 143, 10-12.

Liao J, Zheng Q, Liang X, et al (2013). Effect of fluoroquinolone resistance on 14-day levofloxacin triple and triple plus bismuth quadruple therapy. Helicobacter, 18, 373-7.

Mahachai V, Sirimontaporn N, Tumwasorn S, Vilaichone RK (2011). Sequential therapy in clarithromycin-sensitive and -resistant Helicobacter pylori based on polymerase chain reaction molecular test. J Gastroenterol Hepatol, 26, 825-8.

Marshall BJ, Warren JR (1984). Unidentified curved bacilli in the stomach of patients with gastritis and peptic ulceration. Lancet, 1, 1311-5.

Miehlke S, Krasz S, Schneider-Brachert W, et al (2011). Randomized trial on 14 versus 7 days of esomeprazole, moxifloxacin, and amoxicillin for second-line or rescue treatment of Helicobacter pylori infection. Helicobacter, 16, 420-6.

Parsonnet J, Friedman GD, Vandersteen DP, et al (1991). Helicobacter pylori infection and the risk of gastric carcinoma. N Engl J Med, 325, 1127-31.

Rauws EA, Tytgat GN (1990). Cure of duodenal ulcer associated with eradication of Helicobacter pylori. Lancet, 335, 1233-5.

Srinarong C, Siramolpiwat S, Wongcha-um A, Mahachai V, Vilaichone RK (2014). Improved eradication rate of standard triple therapy by adding bismuth and probiotic supplement for Helicobacter pylori treatment in Thailand. Asian Pac J Cancer Prev, 15, 9909-13.

Vilaichone RK, Mahachai V (2001). Current management of Helicobacter pylori infection. J Med Assoc Thai, 84, 32-38.

Vilaichone RK, Mahachai V, Graham DY (2006). Helicobacter pylori diagnosis and management. Gastroenterol Clin North Am, 35, 229-47. 